\title{
From Systems Change to Systems Changed: Assuming a systems-based approach in response to crisis
}

\author{
Jen Nelles, Tim Vorley and Adam Brown
}

Published in:

Productivity and the pandemic: Challenges and insights from Covid-I9 [ISBN: 978I80037459I] /

edited by Philip McCann and Tim Vorley (Edward Elgar, 202I).

\section{COVID-19: A Shock to the System}

The COVID-19 pandemic is a shock many orders of magnitude greater than anything previously experienced in the UK and around the globe. Even as the public health aspects of the crisis have become more manageable it is clear that the social and economic consequences will be felt for many years to come. Increased productivity has been described as one of the engines of economic recovery after large economic shocks even as the effects of the pandemic have exacerbated longterm weaknesses in the UK (Du, 2020). In a recent analysis from the Productivity Insights Network, Sena (2020) argued that mitigating the economic shock of COVID-19 will require a strong recovery in productivity growth to a level that predates the 2008 Financial Crisis. She also points out that focusing on strengthening productivity growth will be an important source of economic resilience as society adapts until a vaccine is found. Furthermore, with the OECD predicting the UK will face the deepest recession of any nation in the developed world (with a projected economic contraction of II.5-I4\%), maintaining and productivity growth will be vital to managing the downturn and speeding recovery (OECD, 2020).

Growing productivity in the context of a global crisis is likely to be extraordinarily difficult, particularly given that even prior to the pandemic the UK was struggling with puzzlingly low productivity growth. However, such a profound crisis also offers an opportunity - if what we were doing to advance this growth agenda wasn't working before, and everything is in a state of flux, what better time to start afresh?

Such a complex and multifaceted challenge demands a strategy sensitive to these unprecedented conditions. We propose a radically new approach. Or, rather, we argue that we should consider what is, in essence, an old approach - systems thinking - in a more serious manner, and on a larger scale, than ever attempted before. In brief, systems thinking is a set of tools and a framework for exploring problem spaces as (parts of) complex adaptive systems (Mitleton-Kelly, 20I5). This point of view appreciates that much of the social world in which we operate consists of systems - that is, structures of actors, relationships, and rules - characterised by complexity. These systems are more than just complicated. Rather, complexity is a term used to describe systemic attributes such as interdependence between elements, that exhibits nonlinear dynamics, emergent behaviour, and path dependence (Cairney, 2015). In short, this approach views problem spaces as embedded in broader contexts and specifically seeks to explain observed outcomes by searching out interdependencies, interactions, feedback loops, and sources of interference in order to better design (and experiment with) policy. 
This type of approach is particularly apt - and possibly absolutely crucial - for the types of challenges posed by the COVID-I 9 pandemic. It has been, in the most literal sense, a series of shocks to the system. Any coherent attempt to respond must take into account the nature of systemic shocks. Any efforts to intervene in the trajectory of events and affect outcomes must recognise that we are functioning within a system changed. Many old rules no longer apply, the problem space is incredibly dynamic, and success will rely in large part on effectively interpreting and reacting to feedback. To change the system we must accept that we are facing a system that is changed - is changing - and focus on developing the mechanisms to begin to understand what the rules of this evolving system are. As the economic implications of the COVID-19 crisis continue to become apparent this presents an opportunity to explore how the future of the productivity debate might be reimagined from a systems perspective with a focus on future economic resilience.

\section{Complex Systems and Public Policy}

Contemporary theories of complex systems emerged from, is applied in, and draws inspiration from a wide variety of disciplines, including the life sciences, philosophy, cybernetics, and more in addition to public policy [also see Mingers, (20I4); Byrne and Callaghan (20I4); Forrester (2007)]. While there has been considerable cross-fertilisation between disciplines what is notable is that the problems that complexity theory emerged to describe and grapple with transcended disciplines and that they reached, more or less independently, similar conclusions about the nature of the world and the limits of problem solving in that context. In all traditions, theorists struggled with the same fundamental issue that observed outcomes appear to be influenced by a variety of hidden phenomena. These manifest in all sorts of different ways - nonlinearity, randomness, collective dynamics, hierarchy, and emergence - which have been collectively described as "a deck of attributes that have proved ill suited to our intuitive and augmented abilities to grasp and comprehend" (Krakauer, 2019). For students of public policy, these puzzles will be familiar. Too often, carefully designed interventions fail to yield expected results, or they do but create unanticipated consequences. Adopting systems approaches in public policy is one method of uncovering and understanding the "hidden worlds" that influence outcomes.

Systems thinking is distinguished from other approaches to public policy in terms of how it perceives and defines problems and the problem space in which decision makers operate. It distinguished problems that are merely complicated from those that are complex. Complicated problems have causes that can be easily identified and that can be addressed piece-by-piece and for which success is a relatively permanent solution (Poli, 20I3). For instance, building a rocket ship is a complicated problem. It can be addressed in a mechanical fashion - fuel cells can be designed and manufactured separately from guidance systems and assembled later on - and once the rocket is built and functional it will exist until it breaks (i.e. it is a permanent solution). Complex problems, by contrast, emanate from several sources, some of which can be hard to trace, and that interact with one another in unpredictable ways. Complex problems are also dynamic - there is no single permanent solution - and are therefore more accurately managed than solved. Raising a child is often cited as an example of a complex problem. So, too, are the objects of much public policy.

Moreover, systems theorists see the complexity of these problems partly as a function of the complexity of the system(s) within which they exist. As we hinted in the introduction, most social life can be conceptualised as a complex adaptive system. Taking the case of productivity as an example, the economy is a system characterised by complexity. Mitchell (2009: 13) provides a 
standard definition of a complex system as one "in which large networks of components with no central control and simple rules of operation give rise to complex collective behaviour, sophisticated information processing, and adaptation via learning or evolution".

Cairney (2012) outlines six of the most common features of complex systems. To summarise, briefly, the dominant attributes are their interdependence and feedback loops; non-linear relationships; self-organising properties (emergence); and path dependency. Designing effective policy depends on recognising these features of complexity. And, perhaps most importantly, understanding that while systems are made up of components that interact non-linearly the complexity of the system itself is not a function of the components but the result of the interactions between them (Cillers, 2010).

From a systems theory perspective, policy failures stem partly from designs that do not effectively account for these interactions. Often, decision makers are (fairly or not) accused of treating complex problems as if they are merely complicated. For the most part, policy makers inherently understand that systems, and the issues that they want to affect, are complex even if they may not think explicitly in terms of systems or complexity theory. However, decision makers are also organised within compartmentalised bureaucracies, where policy design is governed by logics of best practices, dominated by a dichotomy between evaluation and implementation, and limited by political cycles (Castelnovo and Sorrentino, 2018). Additionally, the principle of bounded rationality argues that it is impossible for any one person within a system to understand the full extent of a system and how it functions (Byrne and Callaghan, 20I4). These, and other, factors result in a tendency for decision makers to seek linear solutions within silos rather than learning the dance of managing complex systems [for further discussion see Poli (20I3); Rhodes et al (20I5); Eppel and Rhodes (2018); Burnes (2005)].

If this is the case, then how can policy makers "learn to dance"? How can decision makers design effective policies when systems theory argues that no one of them can fully comprehend the whole system in which universal rules and assumptions of predictability don't hold? And how indeed can this be accomplished in the tumultuous midst of system altering shocks, such as presented by the COVID-19 pandemic? We will return to these very pressing questions shortly but, first, explore how systems theory treats shocks for some clues to understanding systemic resilience and recovery.

\section{Complexity, system change, and resilience}

Our overall thesis about policy responses to the COVID-19 crisis is this: the magnitude of the disruption to every facet of the economy and society as a result of the pandemic makes adopting a systems approach to recovery particularly vital. When all accepted assumptions, relationships, and axioms have been turned on their heads it is even more important to focus on understanding the new (and evolving mechanisms) and how they function. This is also an opportunity to critically evaluate how we think about the policy making processes and engage in programme design. Exploring how complexity theory understands system shocks and resilience provides a useful framework for thinking about what recovery could, and should, look like and some insights as to how to proceed.

Our choice of title for the introductory section deliberately foreshadows some of the points that we want to highlight here; namely, that amidst dire narratives of economic collapse, crisis, and failure 
what we are really experiencing is system change. While these shocks are certainly acute in some cases, we argue that the economic system overall is resilient. In fact, one of the features of the economy that makes it a complex adaptive system is just that - its propensity for adaptation.

\section{System adaptation and evolution}

A complex system is a series of interconnected elements that collectively execute some function (or functions) in the absence of centralised control. The actors within these systems process feedback and alter behaviour, resulting in a constant process of change, evolution, and learning. Adaptation and change occurs through a variety of routes but most commonly by processing information or reacting to stimulus that kicks off feedback mechanisms. Forrester (1969: 15) famously argued that "systems of information-feedback control are fundamental to all life and human endeavour, from the slow pace of biological evolution to the launching of a satellite". Feedback exists when aggregate patterns form from individual behaviour, and that then individual behaviour responds to those patterns [see Arthur, (20I5)].

The immune systems illustrate an intuitive instance of this process: when attacked by an external pathogen in some cases the body will develop antibodies. This process is based on a feedback loop in which more successful antibodies replicate at greater rates until the most efficient combination, and immunity, is produced. This is an elegant example, but not all types of feedback create such neat results. For instance, individuals in cars combine to form patterns in traffic, to which those individuals respond causing vehicles to alter positions. Feedback loops can also create more dramatic results where they compound and accelerate change in one direction (Anderson and Johnson, 1997): in an example of an ecological feedback loop, warmer temperatures increase microbial activity in soil, which releases more carbon into the atmosphere and contributes to global warming and increasing temperatures, which makes the microbes even more active, and so on. Economies also experience and respond to various types of feedback. In business, order and inventory histories will drive production decisions, affecting available stocks, prices, and delivery timelines, which then drives business inventory decisions. But as Towell (1994) suggests, these processes are not perfect, information lags and delays can sometimes create misalignments that hold systems in suboptimal conditions.

These are just a few of many examples of feedback, adaptation, and learning that can happen within a system. However, the main point, as Towell (1994) suggests, is that studying complexity is ultimately about understanding how systems process feedback - in grasping the formation of structures and how this formation affects the objects causing it. At its heart it is about understanding how systems react to and create change. Significantly, as the above examples demonstrate, these reactions are not always elegant and balancing, but can also be disruptive, imperfect, and messy. This raises an important question - if we accept that the systems that we study are in a constant state of change then what is normal?

\section{What is normal?}

Decision makers and economists often use the term 'equilibrium' to describe a normal or regular state of the economy. However, the principle of constant change that underpins system and complexity theory appear to challenge that perception. In fact, while systems theorists accept that systems can exist in a state that can be described as equilibrium, what constitutes equilibrium is ever 
evolving and is not static or permanent. Rather, it is one of many states and can be achieved in many different ways. Spencer (cited in Byrne and Callaghan, 2014: 92 ), summarises this concept by defining a system as "a whole whose parts are held together by complex forces that are ever rebalancing themselves - $a$ whole whose moving equilibrium is continually disturbed and continually rectified" (emphasis added). Arthur (20I5) even goes so far as to argue that "nonequilibrium is the natural state of the economy, and therefore the economy is always open to reaction", albeit this is a relatively controversial view. Implicit in this approach is a recognition that systemic stability is possible. Crucially, though, a stable system is not a static system.

While it is relatively easy to accept that systems and subsystems exist in a state of constant change it is equally important to recognise that they also tend to function with a degree of stability. In fact, most of the systems that we most frequently study - societies, economies, biological organisms - are relatively stable. Think of the human body. As an organism it is in a state of constant change as it reacts to inputs, growth, and external conditions. As long as everything remains within acceptable parameters, it will continue functioning. Research shows that the human body replaces all of its cells every seven to ten years, but despite this entire replacement of the system it remains your body. As a whole, it continues to function as normal. So what affects system stability is not that change is happening (as it always is) but what kinds of changes occur.

The concept of system function is crucial to understanding system stability and the conditions under which a system will fail. In the simplest terms, systems fail when they are unable to continue their current function. This could prompt the evolution of the system to a different function, or the disappearance of the system completely. Significantly, all of the component elements of a system could remain intact and yet a system could still fail. This is because what is important is not the pieces of the system itself but the relationships and interactions between them. Meadows (2008) summarises this nicely when she states that a system goes on being itself even if elements change as long as interconnections and purposes stay the same. Returning to the concept of equilibrium, Holling (1973), and others since, recognised that systems can shift significantly in structure and composition such that there are multiple possible stable states. Again, the key here is the persistence of system function.

\section{Systems and resilience}

Here we can begin to build some connections between the work of systems theory and scholarship on (specifically, economic) resilience. Interestingly and appropriately, systems approaches were applied early on to the field of resilience studies as an alternative to engineering conceptualisations of resilience that tended to focus on resistance to shocks and ability to (and speed) of return to the pre-shock state (Bohland et al, 2019). Adopting a systems approach, by contrast, defines resilient systems as those that can adapt to sustain their core function(s) following a disruption.

Scholars from a variety of disciplines have engaged with the concept of resilience to understand the behaviour of systems, and vice versa, and derived similar definitions. Building on the work of Walker et al (2004) working in the realm of ecological resilience, Norberg and Cummings (2008:46) define resilience as "the capacity of a system to absorb disturbance and reorganize while undergoing change so as to retain essentially the same function, structure, identity and feedbacks, that is, remain within one regime". Hollings (1973:17), writing decades earlier in the same field, defined it as "the persistence of relationships within a system and is a measure of the ability of these systems to 
absorb changes of state variables, driving variables, and parameters, and still persist", here highlighting the importance of maintaining the character of connections within the system. Writing about resilience in regional economies, Simmie and Martin (2010:3I) identify what has been described as an evolutionary approach to resilience, where it is considered as "an ongoing process rather than a recovery to a (pre-existing or new) stable equilibrium state. This shifts the theoretical analysis from questions about how a system such as an economy is resilient to how it adapts through time to various kinds of stress". While these perspectives are united by several common traits most notably they focus on the elements of a system that can change and those that must persist. Meadows, however, provides an evocative description of another state - a loss of resilience - or, how systems fail:

I think of resilience as a plateau upon which the system can play, performing its normal functions in safety. A resilient system has a big plateau, a lot of space over which it can wander, with gentle, elastic walls that will bounce it back, if it comes near a dangerous edge. As a system loses its resilience, its plateau shrinks, and its protective walls become lower and more rigid, until the system is operating on a knife-edge, likely to fall off in one direction or another whenever it makes a move. Loss of resilience can come as a surprise, because the system is usually paying more attention to its play than its playing space. One day it does something it has done a hundred times before and crashes. (Meadows, 2008: 78)

In this case, system failure is the result of a loss of resilience and the implication is that the source(s) of that weakness are not always obvious. That is, the relationships that allowed the system to function can erode over time in imperceptible ways - until they break. This is a warning that while we tend to think of systems as resilient, or not, there are potentially more nuanced states of system fragility, decline, or consolidation that might be difficult to observe.

Understanding the dynamics of system change, failure, and resilience provides an important foundation for applying systems approaches to responsive policy design in a time of stress and multiple unknowns. Adopting a systems approach in responding to shocks encourages policy makers to focus not just on the metrics, but on the interdependence underlying causes and consequences. In times of economic crisis, particularly those marked by resource contractions, it is tempting to conclude that poor economic outcomes are the results of those resource constraints - i.e. fewer inputs mean fewer outputs. However, what might actually be happening may be far more complex such that aiming policy at increasing inputs may risk treating a symptom while failing to diagnose its core causes. The $s$

As a result of the pandemic, we are likely to see changes to key relationships and dynamics within the system that were once considered axiomatic. If there are aspects of the system that no longer hold or have been disrupted and reconfigured as a result of the crisis, this is what we refer to as systems changed. The change is exogenous to the system and the impact is on the configuration of the system itself. In this scenario, there is a need to rethink the nature of existing and intended policy interventions where the system is different to the previous or intended context.

Together these two approaches provide a conceptual lens to think about how systems thinking can be applied to the way in which we think about policy interventions in the wake of the COVID-19 crisis. Crucially, the systems approach encourages us to identify what parts of systems and 
subsystems are changing (and how) or failing (and why) before proceeding with reactive interventions.

\section{Lessons and prospects for post-pandemic policy}

As this and the other chapters in this edited collection highlight, the impacts of COVID-19 on productivity are profound. The economic, and public health impacts of the pandemic have prompted unprecedented responses from the Government in the UK, and governments around the world. As the other chapters in this volume demonstrate, COVID-I9 has had wide ranging and transformative effects on the UK economy. These impacts are exemplified in different outcomes and impacts on the economy. Webber and Dymski (see Chapter 17), who highlight skyrocketing public expenditures and declining revenues have caused the UK government debt to exceed national GDP for the first time since the 60s. In the labour market, between April and June 2020, Green (see Chapter 8 ) the claimant count more than doubled while job vacancies plummeted, revealing potentially significant trends by region, occupation, age cohort, and sector.

Several contributions also note the incredible uncertainty about spatial impacts. In Chapter I3, Huggins and Thompson point to early evidence and longer-term potential for shifting patterns of urbanisation while in Chapter 12 Gardiner et al. reflect on the potentially profound sectoral and regional challenges that may emerge from the crisis. Docherty et al. (see Chapter II) highlight staggering impacts on public transportation and the potential for longer term impacts on system financing and service levels that may have significant and lasting consequences for mobility. Another recurring theme is the need to consider policy holistically. In Chapter 2, Harris first notes that short term Government responses focused on protecting jobs and mitigating firm closings might have longer term effects on barriers to entry and the effectiveness of resources allocation towards higher-productivity firms. However, these short run measures may be perceived as necessary, but need to be considered in light of other dynamics shifting in the economy. As Henley et al note in Chapter 4, the impact of the pandemic is now of secondary importance compared to what happens next as part of the recovery. The underlying premise of productivity outcomes will, however, remain more important than ever to business, and society more broadly can cannot be considered in a piecemeal way.

As we have argued, the system has shifted and continues to change, and this demands a dynamic and experimental approach of policy. In order to be effective, policy makers need to understand both how the rules of the game and the system are changing in absolute and relational terms. It is only in this way that systems thinking can meaningfully impact economic and societal outcomes by working across traditional policy domains and siloes. This demands a need to understand the relationships among the parts (i.e. policy silos) that comprise the system, rather than the silos themselves. By working in this way there is an opportunity to both leverage and realise the value of interdependencies of policy silos as part of the wider system.

The majority of this chapter serves as a primer on systems approaches to support the case that embracing the complexity of the crisis provides a more nuanced understanding of the nature of shocks and economic resilience. It also serves as a framework to begin applying these concepts to policy design. The principles of systems thinking are particularly critical in the wake of COVID-19 as they provide the basis to reimagine how to approach the long standing but now more acute 
productivity puzzle. The failure to impact productivity through discreet policy interventions highlights the need for an alternative approach. The pandemic has served to demonstrate the complex but highly interdependent nature of the economic and policy system, and it is here that systems thinking can be the basis of an alternative approach.

There is an opportunity in advancing the case for systems thinking in the wake of the pandemic, not least because the priorities of the system, if not the system itself has changed. For policymakers this requires a more holistic perspective, which is in contrast to dominant norms in policy thinking. One of the greatest challenges in applying systems approaches to public policy is the tendency to see (and institutionalise) boundaries that are socially constructed (Meadows, 2008). In pursuing a systems approach it is imperative that policy makers do not fall into the trap of limiting scope of inquiry to their own areas of jurisdiction and control. This may limit both how willing and able policy makers are to perceive and define problems (and their causes), and the interventions they consider possible. This is exemplified in recent study by Nelles et al (2020), which highlights the difficulty of working across policy silos in Northern Ireland, and how policy decisions are subsequently shaped by these artificial divisions.

A key implication of perceiving systems instead of silos is that they are inherently contextual, there are no 'best answers' and 'golden rules' (Castelnovo and Sorrentino, 20I8). While there is still value in policy learning it is important to recognise that policies from other places were developed in a game with (sometimes significantly) different rules. Lessons from elsewhere still play a role, but more effectively as lenses through which to interpret and react to policy feedback. Looking at the experience and policy response of other countries' to COVID-19 and adopting them in the UK without understanding why they (apparently) worked elsewhere is unlikely to succeed.

Together the chapters comprising this edited collection begin to illustrate not only the multifaceted nature of the (post-pandemic) productivity puzzle, but their highly interdependent nature. We contend that as opposed to discrete policy responses that there is a need to work beyond artificial silos, that the pandemic has come to highlight the shortcomings of fragmented policy responses. There is a need for a more joined-up approach to the design and delivery of policy to understand the interdependencies across policy silos. In practice, this is likely to be characterised by more experimental approaches towards policymaking, characterised by greater reflexivity and with feedback through the system leading to better policy design and better policy outcomes.

As this chapter has argued, policy "certainty" is elusive under most circumstances and this is no more apparent than in conditions of crisis, such as the current pandemic. The value of systems thinking is arguably exemplified in the context of crisis, although its value is in its broader adoption and application. The premise is therefore not about maintaining continuity associated with a prepandemic sense of normality, but rather adapting policy responses to the changing economic (and social) realities of the productivity puzzle. In terms of what this means for policymakers, the challenge is threefold:

- First, identifying and defining the contours of the system by mapping the (policy) system can provide an important starting point, although it is not an exact science and needs to be approached as a collaborative exercise (Barbrook-Johnson et al., 2020); 
- Second, policymakers need to understand relationships in the system, as well as the implications and impacts of observed (and hidden) changes. There is also a need to think critically about prioritising policy objectives in what is a dynamic context;

- Third, it is critical that as well as understanding the complexities of policy that there is a willingness and ability to pursue a coordinated response that spans traditional policy domains in more creative and effective ways.

Fundamentally, adopting a systems perspective represents an alternative approach towards policy making and how decisions are made. The pandemic can be seen as an economic and societal inflection point that provides an impetus for change. Systems thinking offers a basis to redress the impacts of the COVID-19 crisis, and protect and enhance resilience by working collaboratively to overcome the prevailing silos of policy thinking. While systems are complex, the adoption of systems thinking in policy need not be complicated. Therefore, while systems-based approaches may be considered unconventional, they are important if we are to move beyond the realms of policy reforms to realise more transformative policy outcomes. 


\section{Bibliography}

Anderson, V. and Johnson, L. (1997). Systems Thinking Basics. From Concepts to Causal Loops. Waltham, Mass Pegasus Communication

Arthur, W.B. (2015) Complexity and the Economy, Oxford: Oxford Economic Press

Barbrook-Johnson, P., Proctor, A., Giorgi, S., and Phillipson, J. (2020). How do policy evaluators understand complexity? Evaluation, 26(3), 315-332. doi:10.1 177//356389020930053

Bohland, J., Davoudi, S. and Lawrence, J. (2019) The Resilience Machine, New York: Routledge.

Burnes, B. (2005). Complexity theories and organizational change. International Journal of Management Reviews, 7(2), 73-90. doi:doi: I 0.1 I I I/j. I468-2370.2005.00107.x

Byrne, D. and Callaghan, G. (2014). Complexity Theory and the Social Sciences: The State of the Art. London: Routledge

Cairney, P. (2012). "Complexity Theory in Political Science and Public Policy." Political Studies Review 10(3): 346-358.

Castelnovo, W. and Sorrentino, M. (2018). Engaging with complexity in a public programme implementation. Public Management Review, 20(7), I0I3-103I. doi:10.1080/14719037.2017.1364406

Cilliers, P. (2010). Difference, Identity and Complexity. In P. Cilliers and R. Preiser (Eds.), Complexity, Difference and Identity (pp. 27-32). London: Springer.

Du, J. (2020). "How to boost UK productivity after coronavirus?" The Conversation https://theconversation.com/how-to-boost-uk-productivity-after-coronavirus-I 33735 Accessed June 29, 2020.

Eppel, E.A. and Rhodes, M.L. (2018). Complexity theory and public management: a 'becoming' field. Public Management Review, 20(7), 949-959. doi:I0.1080/14719037.2017.13644I4

Forrester, J. W. (2007). "System dynamics—the next fifty years." System Dynamics Review 23(2-3): 359-370.

Forrester, J.W. (1969) Industrial Dynamics, MIT Press, Cambridge, MA,

Holling, C.S. (1973). Resilience and stability of ecological systems. Annual Review of Ecology and Systematics 4, I-23.

Krakauer, D. (2019). Introduction. Worlds Hidden in Plain Sight: The Evolving Ideas of Complexity of the Santa Fe Institute, 1984-2019. D. Krakauer. Santa Fe, Santa Fe Institute Press: xxvii-xxix.

Meadows, D. (2008). Thinking in Systems: A Primer. White River Junction, Chelsea Green. 78.

Mingers, J. (2014). Systems Thinking, Critical Realism and Philosophy: A Confluence of Ideas. Abington, Routledge

Mitchell, M. (2009). Complexity: A Guided Tour. Oxford, Oxford University Press.

Mitleton-Kelly, E. (20I5) Effective policy making: addressing apparently intractable problems' in Geyer, R. and Cairney, P. (eds) Handbook on Complexity and Public Policy, Edward Elgar, p.III-I 28

Nelles, J., Brown, A. and Vorley T. (2020) " Mapping the Cognitive Landscape of Productivity in Northern Ireland: A Systems Approach to Understanding Productivity Policy' Productivity Insights Network https://productivityinsightsnetwork.co.uk/app/uploads/2020/07/JNTVABReport_ExecSummaryOnly.pdf/ Accessed July 29, 2020.

Norberg, J. and Cumming, G (2008) Complexity theory for a sustainable future, New York, Columbia University Press 
OECD (2020). The world economy on a tightrope. OECD Economic Outlook (June 2020). Paris, OECD.

Poli, R. (20/3). A Note on the Difference Between Complicated and Complex Social Systems. Cadmus, 2(I), I42-I47.

Rhodes, M.L., Murphy, J., Muir, J., and Murray, J. A. (2015). Public Management and Complexity Theory: Richer Decision-Making in Public Services. London: Routledge.

Sena, V. (2020). "Does productivity still matter?" Productivity Insights Network https://productivityinsightsnetwork.co.uk/2020/05/does-productivity-still-matter/ Accessed June 29, 2020.

Simmie, J. and Martin, R. (2010) The economic resilience of regions: towards an evolutionary approach, Cambridge Journal of Regions, Economy and Society, 3 (I) 27-43, https://doi.org// 0.1093/cjres/rsp029.

Towell, D (1994) Industrial Dynamics Modelling - Not So Much a Subject, More a Way of life, Measurement and Control, 27(7), 226-23I. https://doi.org/I 0.I I77/002029409402700705

Walker, B.H, Holling,C.S. Carpenter, S.R. and Kinzig, A. (2004) . Resilience, adaptability and transformability in social-ecological systems. Ecology and Society 9(2):5 